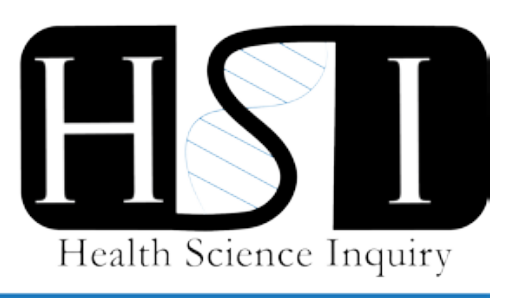

\title{
A fine balance: the complex interplay between host and microbes in inflammatory bowel disease
}

\author{
Emma Brownlie \\ Department of Molecular Genetics, University of Toronto
}

Inflammatory bowel disease (IBD) is one of the most common chronic inflammatory disorders, with millions of people suffering from this debilitating condition [1]. It is characterized by perturbed intestinal barrier function and an infiltration of immune cells beyond the intestinal wall [2]. It is responsible for high levels of morbidity in the form of recurrent bouts of abdominal pain and diarrhea, and an increased risk of severe complications such as colon cancer and fistula [2]. The prevalence of this disorder has increased over the past decade, and this trend has been predicted to continue worldwide $[1,3]$. However, the etiology of IBD is multifactorial, encompassing genetic, immunological, and environmental components, which has made pinning down causes and designing treatments especially difficult. An environmental component that particularly complicates this equation is the microbiota.

The human gastrointestinal tract is colonized by trillions of microbes, outnumbering our own cells and forming a complex community that we refer to as the gut microbiota. The colon is particularly densely populated, containing over 100 billion microbes per gram and supporting hundreds of different bacterial species [4]. Together, these organisms are able to influence many aspects of host physiology, for example metabolizing nutrients, modulating the immune system, and maintaining the integrity of the gut mucosal barrier. Generally the microbiota interacts symbiotically with the host to maintain a homeostatic state in the gut, and in a healthy adult the microbiota is generally stable and robust to perturbation [5]. However, major shifts in the gut microbiota have been shown to play a significant role in a number of diseases, one of the most prominent being IBD.

In the case of IBD, these alterations are commonly associated with an initial environmental stimulus that triggers inflammation and a shift in the gut microbiota. In healthy individuals, the immune system balances on a knife edge: it dampens the inflammatory response to beneficial resident microbes, while remaining poised to respond to external insults. These insults can include antibiotics, drugs, diet, toxins, and microbial pathogens all of which can be risk factors for the development of IBD [6]. Any

one or a combination of these factors can trigger activation of the immune system, transiently provoking inflammation and disruption of the epithelial barrier. In healthy individuals, the resident microbes aid in the maintenance of this homeostasis, exerting a protective effect against development of colitis. This is done through the induction of anti-inflammatory factors; even a limited selection of Clostridia strains from the human gut microbiota has been shown to be able to induce the production of regulatory $\mathrm{T}$ (Treg) cells, interleukin-10 (IL-10), and transforming growth factor beta 1 (TGF- $\beta 1$ ) [7]. In a proportion of individuals, however, the inflammation engendered by the transient stimulus persists, leading to the development of chronic IBD. This maladaptive response is associated with profound and sustained modifications to the microbiota, and a disruption of the symbiotic relationship between host immune system and gut microbes.

The progression of disease is accompanied by the dominance of microbial groups not usually prevalent in the healthy gut. Changes in microbiota composition vary on an individual basis, but usually include a significant increase in abundance of members of the Enterobacteriaceae and Fusobacteriaceae families [8]. This is commonly accompanied by a corresponding decrease in abundance of Clostridia, Bifidobacteriaceae, Ruminococcaceae, and Faecalibacterium, as well as an overall loss of bacterial species diversity [8]. This set of bacteria tends to induce a tolerogenic immune response and mediate anti-inflammatory activity [9]. Ultimately what these alterations boil down to is a shift in the microbiota, favouring microbes that thrive under conditions of inflammation, and serve to further activate the immune response. This leads to a vicious cycle of interactions between the gut microbiota and the host, where inflammation drives a shift in the prevalence of microbes, which triggers more inflammation and further promotes the growth of inflammation-inducing microbes. Consequently, the question becomes: how can we break this cycle? A number of approaches have been used to treat IBD, including 5-aminosalicylate (5-ASA) agents, corticosteroids, immunomodulators, and surgical intervention, but none have proven curative or even 
consistently efficacious [8]. Newer treatments involving prebiotics, probiotics, and fecal microbiota transfer have also met with inconsistent success. All of these treatments are complicated by the dramatic variation in microbiota composition and genetics between individuals, making it difficult to even define a 'healthy' versus a 'diseased' microbiota [10]. The severity and increasing prevalence of IBD highlights the need for further study to understand the key contribution of the microbiota in the progression of this disease. The current treatment outcomes indicate a need to take into consideration the interaction of the gut microbiota with host genetic and immunological factors on an individual basis to shed light on this question.

\section{References}

[1] Kaplan, G. G. (2015) The global burden of IBD: from 2015 to 2025. Nat Rev Gastroenterol Hepatol., 12, 720727.

[2] Abraham, C., Cho, J. H. (2009) Inflammatory Bowel Disease. N Engl J Med., 361, 2066-2078.

[3] Rohr, M., Narasimhulu, C. A., Sharma, D., Doomra, M., Riad, A., Naser, S., Parthasarathy, S. (2018) Inflammatory diseases of the gut. J Med Food., 21 (2), 1-14.

[4] Sender, R., Fuchs, S., Milo, R. (2016) Revised estimates for the number of human and bacterial cells in the body. PLoS Biol., 14 (8), e1002533.
[5] Knight, R., Parfrey, L. W. (2012) Spatial and temporal variability of the human microbiota. Clin Microbiol Infect., 18 (4), 5-7.

[6] Dubeau, M. F., lacucci, M., Beck, P. L., Moran, G. W., Kaplan, G. G., Ghosh, S., Panaccione, R. (2013) Drug-induced inflammatory bowel disease and IBD-like conditions. Inflamm Bowel Dis., 19 (2), 445-456.

[7] Atarashi, K., Tanoue, T., Oshima, K., Suda, W., Nagano, Y., Nishikawa, H., Fukuda, S., et al. (2013) Treg induction by a rationally selected mixture of Clostridia strains from the human microbiota. Nature, 500, 232-236.

[8] Eom, T., Kim, Y. S., Choi, C. H., Sadowsky, M. J., Unno, T. (2018) Current understanding of microbiota and dietary therapies for treating inflammatory bowel disease. J Microbiol., 56 (3), 189-198.

[9] Rajca S, Grondin V, Louis E, Vernier-Massouille G, Grimaud JC, Bouhnik Y, Laharie D et al. Alterations in the intestinal microbiome (dysbiosis) as a predictor of relapse after infliximab withdrawal in Crohns disease. Inflamm Bowel Dis., 2014;20(6):978-986.

[10] Hand, H. W., Vujkovic-Cvijin, I., Ridaura, V. K., Belkaid, Y. (2016) Linking the microbiota, chronic disease and the immune system. Trends Endocrinol Metab., 27 (12), 831-843.

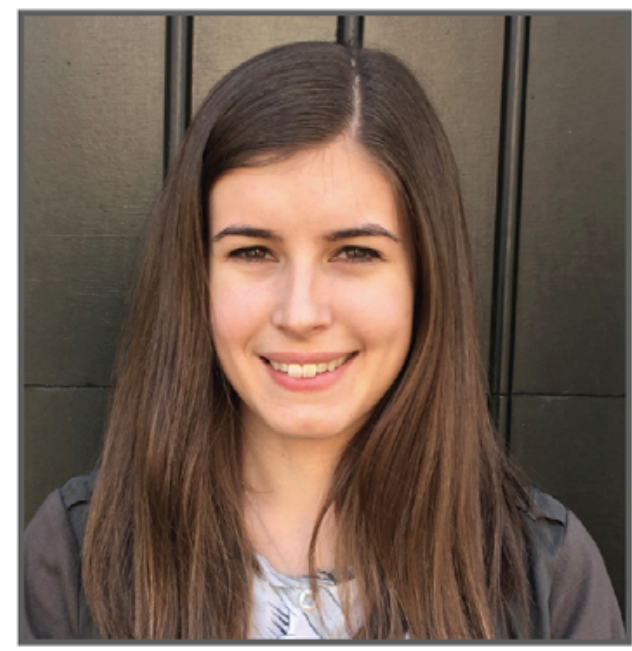

Emma is a second year MSc student at the University of Toronto in the department of Molecular Genetics. While completing a BScH at Queen's University, she spent summers working at the biotechnology firm DNA Genotek, where she became interested in studying the microbiota. Her current research focuses on developing and utilizing in vitro models to examine bacterial community dynamics of the mouse gut microbiota. 